\title{
Arabidopsis thaliana Embryo Sac Mitochondrial Membrane Potential Stain
}

María Victoria Martin ${ }^{1}$, Diego Fernando Fiol ${ }^{2}$, Eduardo Julián Zabaleta ${ }^{3}$ and Gabriela Carolina Pagnussat $^{3^{*}}$

${ }^{1}$ Developmental and Mitochondrial Biology Department, National University of Mar del Plata , Mar del Plata, Argentina ; ${ }^{2}$ Plant Physiology Department, National University of Mar del Plata , Mar del Plata, Argentina; ${ }^{3}$ Developmental and Mitochondrial Biology Department, National University of Mar del Plata, Mar del Plata, Argentina

*For correspondence: gpagnussat@mdp.edu.ar

[Abstract] The aim of this experiment is to study mitochondrial functional status in Arabidopsis embryo sacs using the membrane potential indicator JC-1. Changes in the membrane potential are presumed to be due to the opening of the mitochondrial permeability transition pore (MPTP), allowing passage of ions and small molecules. The resulting equilibrium of ions leads in turn to the decoupling of the respiratory chain and the release of cytochrome $\mathrm{c}$ into the cytosol, a distinctive feature of the early stages of programmed cell death.

JC-1 is a lipophilic dye that can selectively enter into mitochondria and reversibly change color from green to red as the membrane potential increases. In healthy cells with high mitochondrial potential, JC-1 spontaneously forms complexes with intense red fluorescence. On the other hand, in mitochondria with low mitochondrial potential, JC-1 remains in the monomeric form, which exhibits only green fluorescence (Martin et al., 2013; Hauser et al., 2006).

This protocol could be used in isolated mitochondria, and in a variety of cell types and different tissues of plants and other organism.

\section{Materials and Reagents}

1. Flowers at different developmental stages from an Arabidopsis inflorescence

2. JC-1 Dye (Life Technologies, Molecular Probes ${ }^{\circledR}$, catalog number: T3168)

3. DMSO 99.9\% (Sigma-Aldrich, catalog number: D8418)

4. Stock solution (10 mg.ml $\mathrm{ml}^{-1}$ of $\mathrm{JC}-1$ in DMSO)

5. Working solution (10 ug. $\mathrm{ml}^{-1}$ of $\mathrm{JC}-1$ in buffer $\mathrm{A}$ )

6. Buffer A (20 mM HEPES buffer, pH 7.2) (Sigma-Aldrich, catalog number: H3375) (see Recipes)

\section{Equipment}


1. Confocal microscope (Nikon Eclipse C1 Plus Confocal microscope, using EZ-C1 3.80 imaging software and Ti-Control)

2. Dissecting microscope (Nikon Corporation, model: SMZ800)

3. Coverslip ( $18 \times 18 \mathrm{~mm})$

4. Microscopic slide $(26 \times 76 \mathrm{~mm})$

5. $1 \mathrm{ml}$ insulin syringe with the $0.3 \times 13 \mathrm{~mm}$ needle $(\mathrm{BD})$

6. Needle point tweezers

\section{Software}

1. NIH Image J software 1.47 for Windows (http://rsb.info.nih.gov/ii/)

\section{Procedure}

1. Pistils isolation (Figure 1, A-E).

Using a pair of tweezers, take flowers, at different developmental stages from an Arabidopsis inflorescence (Alvarez-Buylla et al., 2010). On a microscopic slide and under a dissecting microscope, remove sepals, petals, anthers and stigma using the needles of two $1 \mathrm{ml}$ insulin syringes $(0.3 \times 13 \mathrm{~mm})$ and make longitudinal superficial cuts on pistils at each side of the septum (Figure 1).

2. Pistils stain (Figure 1, F-G).

a. Submerge the pistils in $100 \mu \mathrm{l}$ of a solution containing $10 \mu \mathrm{g} \cdot \mathrm{ml}^{-1}$ of JC-1 in buffer A.

b. Incubate for $30 \mathrm{~min}$ at room temperature without shaking. Protect from light, as the dye is photosensitive.

c. Gently wash the pistils three times with buffer A.

3. Sample preparation for microscopy (Figure 1, H-L).

a. On a microscopic slide under a dissecting microscope, use the needles of two $1 \mathrm{ml}$ insulin syringes $(0.3 \times 13 \mathrm{~mm})$ to dissect the pistils exposing the ovules

b. Add a drop of buffer $A$ and cover the sample with a coverslip.

c. Immediately observe under a confocal microscope. The intensities of green (excitation/emission wavelength $=485 / 538 \mathrm{~nm}$ ) and red (excitation/emission wavelength $=485 / 590 \mathrm{~nm}$ ) are analyzed. 


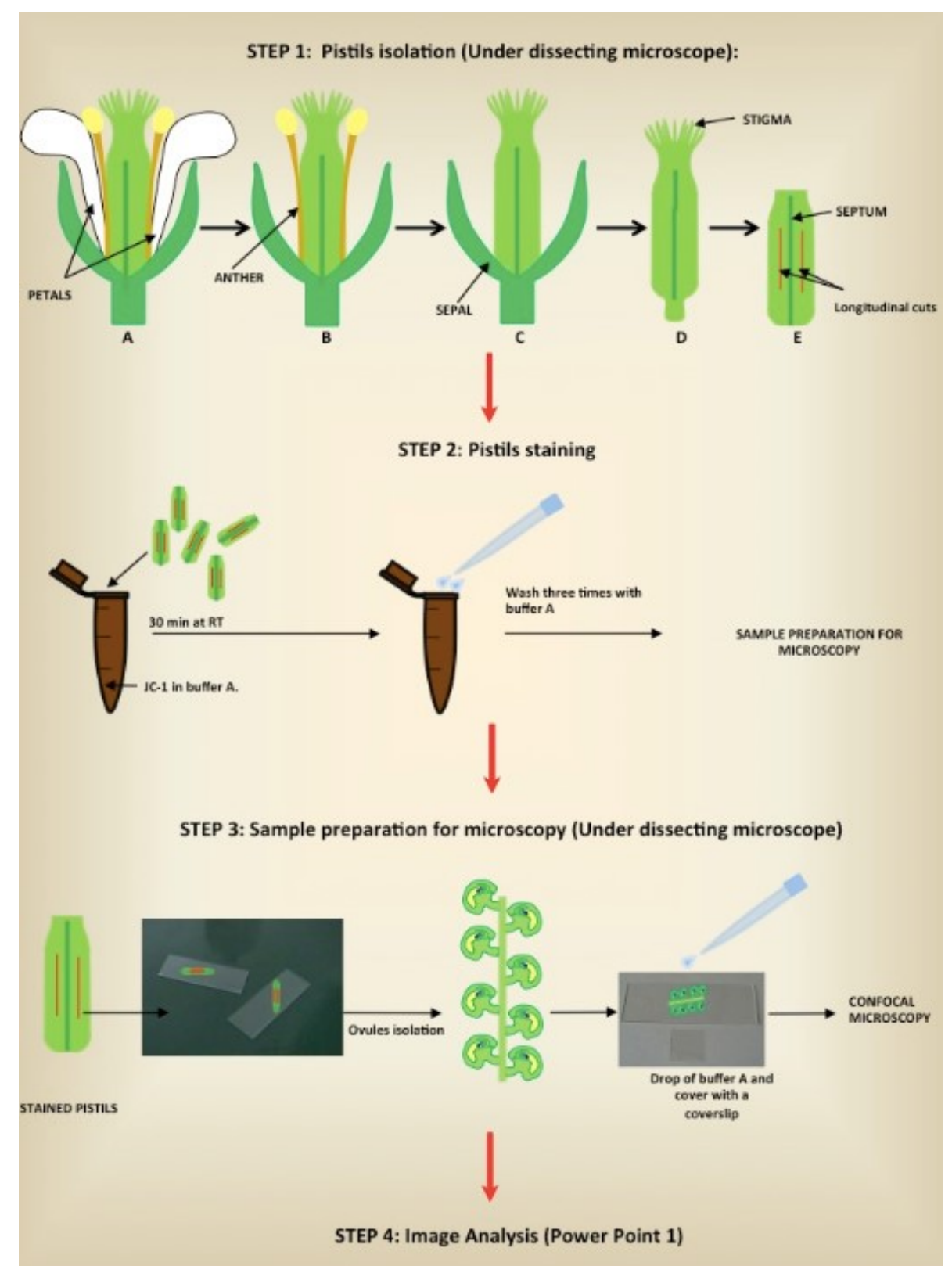

Figure 1. Schematic illustration showing the steps required for embryo sac mitochondrial staining

4. Image analysis (Power Point 1). The ratio of red to green fluorescence of JC-1 images is calculated using NIH Image $\mathrm{J}$ software.

a. The outline of each embryo sac is delimited using the freehand tool to create a region of interest (ROI) and saved using the ROI manager tool (Analyze>tools>ROI manager>Add). 
b. In the "Analyze" menu, select "set measurements" and click on area and "mean gray value".

c. Using the image Menu, select "color" and then "split channels".

d. Close the image in the blue channel. On the green channel, go to the analyze>tools>ROI manager and select the ROI added before. The ROI will appear on the image. Still in the ROI manager go to more>multi Measure. The result will appear in a new window.

e. Repeat this step with the image in the red channel.

f.Copy the results and paste them in an excel worksheet.

g. Calculate the red to green fluorescence ratio for each ROI.

\section{$\underline{\text { Recipes }}$}

1. Buffer $A$

20 mM HEPES buffer ( $\mathrm{pH} 7.2)$

For $1 \mathrm{~L}$ of $1 \mathrm{M}$ HEPES buffer:

Dissolve $238.3 \mathrm{~g}$ HEPES (free acid) in $500 \mathrm{~mL}$ of ddH2O

Stir while adjusting the $\mathrm{pH} 7.2$ with $0.5 \mathrm{~N} \mathrm{NaOH}$

Bring up the volume to $1 \mathrm{~L}$ with $\mathrm{ddH}_{2} \mathrm{O}$ to prepare $1 \mathrm{~L}$ of $20 \mathrm{mM}$ HEPES buffer (Buffer $\mathrm{A}$ )

Add $20 \mathrm{ml}$ of $1 \mathrm{M}$ HEPES buffer in $980 \mathrm{ml}$ of $\mathrm{ddH}_{2} \mathrm{O}$

\section{Acknowledgments}

This protocol was adapted from Hauser et al., 2006. This work was supported by The Howard Hughes Medical Institute (HHMI), National Scientific and Technical Research Council (CONICET), National Agency for Promotion of Science and Technology (AGENCIA) and National University of Mar del Plata (UNMdP). We are grateful to the Editorial Committee of Bio-protocol for kindly inviting us to write this protocol.

\section{$\underline{\text { References }}$}

1. Alvarez-Buylla, E. R., Benitez, M., Corvera-Poire, A., Chaos Cador, A., de Folter, S., Gamboa de Buen, A., Garay-Arroyo, A., Garcia-Ponce, B., Jaimes-Miranda, F., PerezRuiz, R. V., Pineyro-Nelson, A. and Sanchez-Corrales, Y. E. (2010). Flower development. Arabidopsis Book 8: e0127. 
2. Hauser, B. A., Sun, K., Oppenheimer, D. G. and Sage, T. L. (2006). Changes in mitochondrial membrane potential and accumulation of reactive oxygen species precede ultrastructural changes during ovule abortion. Planta 223(3): 492-499.

3. Martin, M. V., Fiol, D. F., Sundaresan, V., Zabaleta, E. J. and Pagnussat, G. C. (2013). oiwa, a female gametophytic mutant impaired in a mitochondrial manganese-superoxide dismutase, reveals crucial roles for reactive oxygen species during embryo sac development and fertilization in Arabidopsis. Plant Cell 25(5): 1573-1591. 